\title{
Center-limb dependence of photospheric velocities in regions of emerging magnetic fields on the Sun (Research Note)
}

\author{
A. Khlystova \\ Institute of Solar-Terrestrial Physics, Siberian Branch of the Russian Academy of Siences, 664033 Irkutsk, Russia \\ e-mail: hlystova@iszf.irk.ru
}

Received 16 September 2010 / Accepted 10 January 2011

\section{ABSTRACT}

\begin{abstract}
Aims. We investigate the ratio of the vertical and horizontal velocities of the photospheric plasma flows in the region of emerging magnetic fields on the Sun.

Methods. We carried out a study of photospheric velocities during the first hours of the appearance of 83 active regions with magnetic flux more than $10^{21} \mathrm{Mx}$ with data acquired by the Michelson Doppler Imager (MDI) on board the Solar and Heliospheric Observatory (SOHO). The emerging magnetic fluxes under investigation were isolated from extended concentrations of existing magnetic fields; they have different spatial scales and are located at different distances from the solar disk center.

Results. We found that the values of maximum negative Doppler velocities that accompany the emergence of active region magnetic fields during the first $12 \mathrm{~h}$ increase nonlinearly with the heliocentric angle. This result shows that the horizontal photospheric velocities of plasma outflows are higher than the vertical ones of the plasma upflows during the first hours of the emergence of active regions. The horizontal velocity component at the site of emerging active regions exceeds that of convective flows in the quiet Sun. A comparison between the velocities and the maximum value of the total magnetic flux has not revealed any relation.
\end{abstract}

Key words. magnetic fields - Sun: photosphere - Sun: activity

\section{Introduction}

The emerging magnetic flux of active regions on the solar surface appears in the form of separate loops; they then coalesce to form opposite polarity poles that separate and increase in size (Strous \& Zwaan 1999). Earlier studies of velocities of plasma motions were carried out in the region of emerging magnetic fields at different height levels of the Sun. Investigations into the chromospheric level have been made by Bruzek $(1967,1969)$ and others (see review by Chou 1993). Velocities at the photospheric level were measured with different methods (discussed in more detail below). In the last decade, helioseismological methods have been applied to study the subphotospheric level (Chang et al. 1999; Kosovichev et al. 2000; Zharkov \& Thompson 2008; Kosovichev 2009; Komm et al. 2008, 2009).

Direct measurements of photospheric velocities revealed negative values (blue Doppler shift) on the polarity inversion line of emerging magnetic fields. The events under consideration were located in the central part of the solar disk, and, therefore, vertical motions were measured. Negative velocities or plasma upflows up to $1 \mathrm{~km} \mathrm{~s}^{-1}$ were observed in separate magnetic loops inside emerging active regions (Tarbell et al. 1990; Lites et al. 1998; Strous \& Zwaan 1999; Kubo et al. 2003; Grigor'ev et al. 2009). Grigor'ev et al. (2007) revealed high velocities of about $1.7 \mathrm{~km} \mathrm{~s}^{-1}$ at the beginning of the powerful active region emergence NOAA 10488 at heliographic coordinates N08 E31 $\left(B_{0}=+4.9\right)$.

Indirect measurements of the horizontal velocities of the photospheric flow that accompanies the emergence of active regions were made by tracking the displacement of individual magnetic elements. The velocities obtained ranged from 0.1 to
$1.4 \mathrm{~km} \mathrm{~s}^{-1}$ (Frazier 1972; Schoolman 1973; Strous \& Zwaan 1999). Barth \& Livi (1990) studied 45 bipolar pairs in the emerging active region. The opposite polarity poles were separating from each other with velocities of $0.5-3.5 \mathrm{~km} \mathrm{~s}^{-1}$, decreasing with time (consequently, the drift with regard to the polarity inversion line is only half that value). Grigor'ev et al. (2009) calculated the separation velocities of the external boundaries of the photospheric magnetic flux in the active region NOAA 10488. The velocities decreased as the magnetic fields emerged: they were $2-2.5 \mathrm{~km} \mathrm{~s}^{-1}$ by the end of the first hour and $0.3 \mathrm{~km} \mathrm{~s}^{-1}$ in two hours and a half.

It should be noted that there are several papers dealing with the study of vertical (Guglielmino et al. 2006) and horizontal (Harvey \& Martin 1973; Chou \& Wang 1987; Hagenaar 2001) velocities at photospheric level during the appearance of ephemeral active regions. We do not discuss these works in detail, since they concern another spatial scale of emerging magnetic fields.

The papers above are the researches into photospheric velocities that accompany the emergence of magnetic fields in active regions located only in the central zone of the solar disk. Plasma motions in the emerging magnetic fields distant from the disk center have never been studied before.

\section{Data processing and investigated objects}

We used full solar disk magnetograms and Dopplergrams in the photospheric line Ni I $6768 \AA$ and continuum images obtained on board the space observatory SOHO/MDI (Scherrer et al. 1995). The temporal resolution of the magnetograms and 

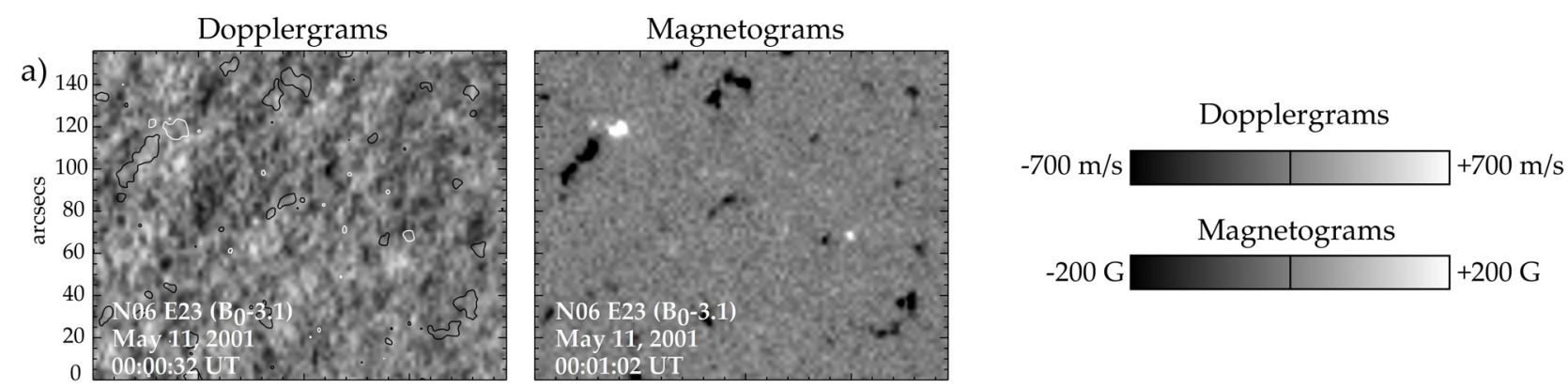

Calculation of the maximum negative velocity
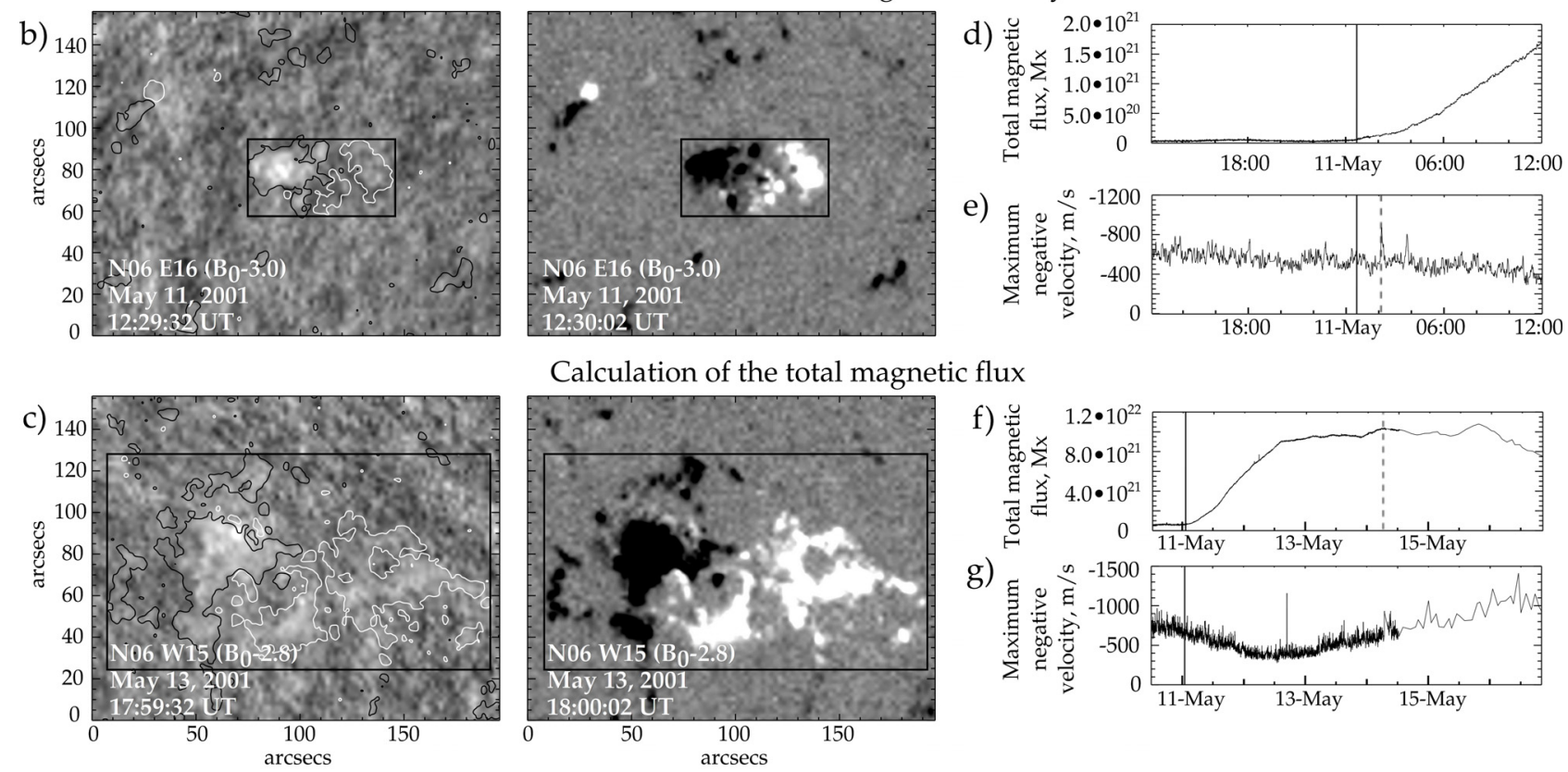

Calculation of the total magnetic flux
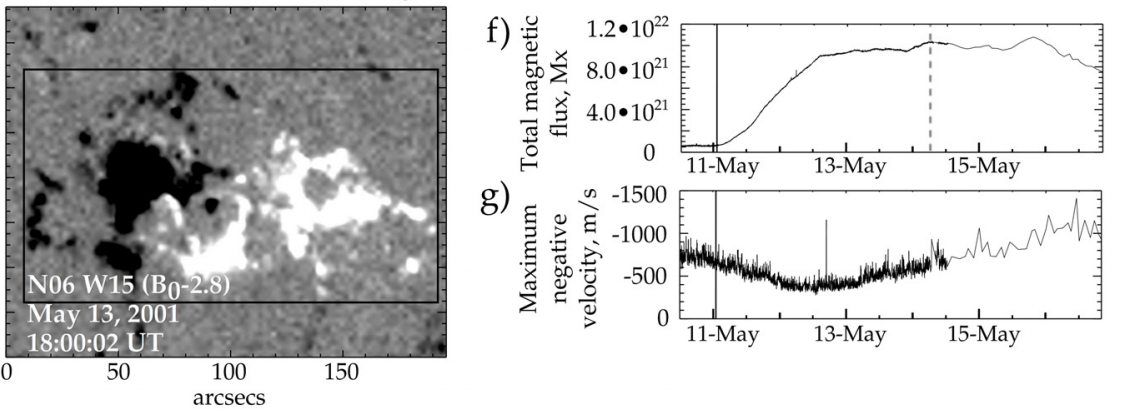

Fig. 1. Active region NOAA 9456: a) the solar surface $30 \mathrm{~min}$ before the magnetic field emergence; b) $12 \mathrm{~h}$ after the beginning of the emergence; c) near the maximum of evolution. The left column represents Dopplergrams with magnetic field isolines $+60 \mathrm{G}$ (white line) and $-60 \mathrm{G}$ (black line); the positive velocities correspond to the motion of matter away from the observer, while the negative velocities correspond to the motion of matter toward the observer. The right column represents magnetograms of the longitudinal field; the black color corresponds to the negative magnetic field, while the white one corresponds to the positive magnetic field. The small rectangle marks the calculation area of the maximum negative velocity during the first $12 \mathrm{~h}$ of the magnetic field emergence; the big one indicates the calculation area of the total magnetic flux. Plots: d), e) variation in the parameters investigated during the first $12 \mathrm{~h}$ of the magnetic field emergence; f), g) variation in the parameters investigated until the maximum of the active region evolution. The beginning of the active region appearance is marked by the vertical solid line; the extreme values of the maximum negative velocity in plot $\mathbf{e}$ ) and the total magnetic flux in plot $\mathbf{f}$ ) are shown by the vertical dashed line.

Dopplergrams is $1 \mathrm{~min}$, that of the continuum, $96 \mathrm{~min}$. The spatial resolution of the data is $4^{\prime \prime}$, the pixel size is approximately $2^{\prime \prime}$. Magnetograms with a 1.8 .2 calibration level were used (Ulrich et al. 2009). Besides photospheric velocities of solar plasma motions, the Dopplergrams include the contribution of 1) the velocity of the differential solar rotation; 2) the velocity of the SOHO satellite with regard to the Sun; 3) the instrumental distortions caused by nonuniform transmission of MDI's filter systems on the field of view. The technique described in Grigor'ev et al. (2007) was used to separate photospheric velocities. The negative velocity on SOHO/MDI Dopplergrams corresponds to the blue Doppler shift (motion of matter toward the observer); the positive one, to the red Doppler shift (motion of matter away from the observer). There are regular errors in velocity measurements at different distances from the disk center. For example, Doppler velocities near the limb are underestimated by up to $40 \mathrm{~m} \mathrm{~s}^{-1}$ because of limb darkening (Albregtsen $\&$ Andersen 1985). Additionally, we deal with different height levels above the solar surface in measurements of velocities at the disk center and near the limb because of the different optical thickness of the atmosphere.
We study the velocities that accompany the emerging magnetic fields. Therefore, the precise spatial superimposition of the used data is vary important. For this purpose, the region of the emerging magnetic fields was taken from the time sequences of magnetograms and Dopplergrams, taking into account solar rotation. An approximate value of the displacement of the region was calculated with the differential rotation law for photospheric magnetic fields (Snodgrass 1983). The exact tracking of the region under investigation was performed by applying two magnetograms adjacent in time, with the use of cross-correlation analysis. This procedure requires the existence of the magnetic poles which slightly vary in time on the magnetograms. The heliographic coordinates obtained were used to crop identical fragments with a size of $160^{\prime \prime} \times 160^{\prime \prime}$ (or $80 \times 80$ pixels) from the magnetogram and Dopplergram obtained at the same time. Thus, we achieved the precise spatial superimposition of the data. It has allowed us to draw reliable conclusions about the processes that take place. For the active region emerging near the limb, we selected the cropped region in a way that it excludes the area outside the limb. 
Time variations of the total magnetic flux of the active region and the maximum negative velocities were calculated with the use of a sequence of the magnetogram and Dopplergram fragments corresponding to the emerging active region. The calculation area was limited to the region of the emerging magnetic fields (Fig. 1). The position of the limits was visually controlled.

The plasma motions connected with the emerging magnetic fields are observed on the background of the convective flows. If the average velocity or the velocity flux is considered as a characteristic of motions of matter, the center-limb dependence of convective velocities will make a substantial contribution. Besides, the active regions under consideration have different spatial scales and are located at different distances from the solar disk center; therefore the solar surface regions where the calculation is performed have different sizes. This complicates a comparison of the emerging active regions that are located at different distances from the disk center. The maximum negative velocity (motion of matter toward the observer) is taken as a characteristic of plasma motion in the regions of the emerging magnetic fields during the first $12 \mathrm{~h}$ (Fig. 1b). The magnetic field emergence begins with the appearance of the loop apex where the magnetic field is horizontal; therefore, we began the calculation of the maximum velocity values $30 \mathrm{~min}$ before the beginning of the appearance of active region. The magnetic flux of the active region emerges as separate loops, and the maximum negative velocity corresponds to the appearance of a single magnetic loop. Therefore, the chosen parameter does not characterize the emerging active region as a whole, it only shows the highest velocity. For example, two peaks corresponding to the emergence of two different magnetic loops can be distinguished in the plot of variations in the maximum negative velocity during the first hours of the NOAA 9456 appearance (Fig. 1e). The residual magnetic flux emerges at lower velocities. To exclude Evershed flows we also took care of the time of the penumbra formation in sunspots, using continuum images. The Evershed flows are horizontal photospheric outflows in a sunspot penumbra. Their maximum velocity values can reach $2 \mathrm{~km} \mathrm{~s}^{-1}$ in SOHO/MDI low spatial resolution data (Bai et al. 1998).

The total magnetic flux is calculated inside isolines $\pm 60 \mathrm{G}$ taking into account the projective effect (Fig. 1c)

$\Phi=\left|\sum_{i=1}^{n_{+}}\left(B_{i} \cdot S_{i}\right)\right|+\left|\sum_{i=1}^{n_{-}}\left(B_{i} \cdot S_{i}\right)\right|$,

where $\Phi$ is the total magnetic flux in $\mathrm{Mx}, B_{i}$ is the magnetic field induction in $\mathrm{G}, S_{i}$ is the area on the solar surface corresponding to $i$ th pixel, $n_{+}$is the number of pixels inside isoline $+60 \mathrm{G}$ (positive polarity) and $n_{-}$is the number of pixels inside isoline $-60 \mathrm{G}$ (negative polarity). The isoline level of $60 \mathrm{G}$ was chosen to exclude both the magnetogram noise (about $30 \mathrm{G}$ ) and the contribution of the magnetic field with a short lifetime. Sometimes small magnetic loops appear in the place of the future active region several hours prior to the main emergence of the magnetic fields. The beginning of the active region emergence was taken as a time moment corresponding to the beginning of the continuous growth of magnetic flux. We determined the maximum value of the total magnetic flux by the first inflection point of the curve where the flux increase was followed by its decrease (Fig. 1f) or by the last value of the plot (for active regions passing beyond the west-limb or with insufficiently downloaded data sequences). Note that powerful active regions significantly increase in size as they develop, and the extended concentrations of the magnetic fields existing on the surface enter into the region of new flux

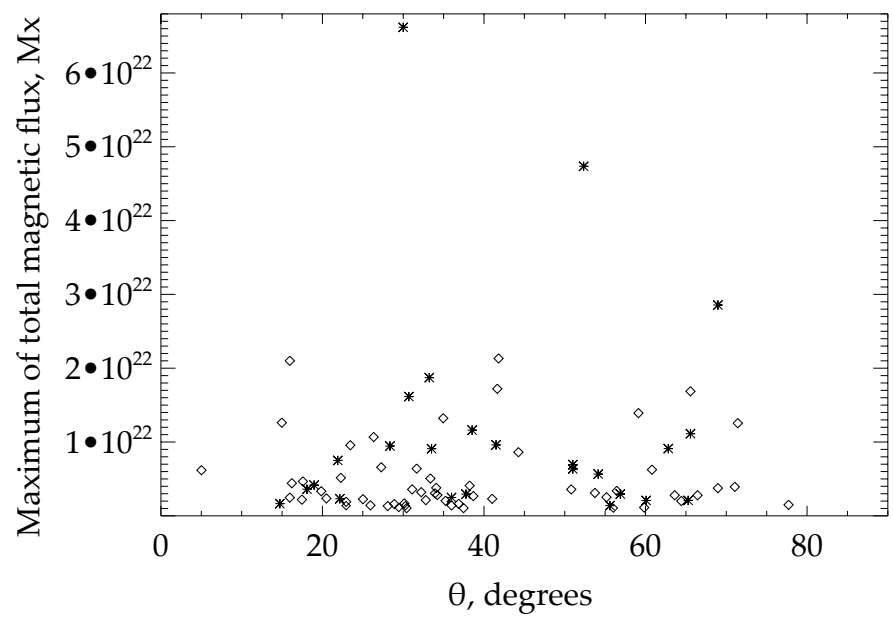

Fig. 2. Total magnetic flux of the active regions under investigation versus the heliocentric angle that corresponds to the beginning of the magnetic field emergence. The total magnetic flux maximum was determined by the inflection point in the increase of the flux curve (marked by diamonds) or by the last measurement (marked by asterisks). Active regions with magnetic saturation are also marked by asterisks. In active regions marked by asterisks, the maximum of total magnetic flux may be higher.

emergence (Fig. 1a-c). Interaction between existing and emerging magnetic fields is accompanied by integration or cancellation processes and, hence, by increase or decrease in the magnetic flux. In order to take this into account to some extent, the signal background that existed before the emergence of magnetic fields was subtracted from the total magnetic flux maximum in all active regions.

The selection of active regions for this investigation was based on the following criteria. Active regions have to emerge on the visible side of the solar disk. Emerging magnetic fields have to be isolated from the extended concentrations of existing magnetic fields. The presence of single poles with a total magnetic flux of less than $0.5 \times 10^{21} \mathrm{Mx}$ was allowed in the area of the direct emergence of an active region during the first $12 \mathrm{~h}$. The objects under investigation have to have complete data series with a one-minute time resolution during the first $12 \mathrm{~h}$ of appearance. With this in mind, the 83 active regions were selected for the period of 1999-2008. Among them, there were 80 active regions with a normal magnetic field configuration and three with an reverse magnetic field configuration according to Hale's polarity law. The events had different spatial scales and were located at different distances from the solar disk center (Fig. 2). The total magnetic flux of each event exceeded $10^{21} \mathrm{Mx}$. Magnetic saturation is possible in SOHO/MDI measurements of the magnetic field strength. This occurs when the spectral line is shifted out of the filter system passband. Liu et al. (2007) modeled MDI measurements and demonstrated that magnetic saturation takes place only in strong magnetic fields with high velocities. According to their calculations, magnetic saturation is reached at the magnetic field strength of $2800 \mathrm{G}$, when the velocity is $\pm 2000 \mathrm{~m} \mathrm{~s}^{-1}$. In our study, the maximum magnetic field strength exceeds $2800 \mathrm{G}$ only in two events of the active regions under consideration. These events are also marked by asterisks in Fig. 2. The morphology of the active regions (i.e., the degree of fragmentation of magnetic fields, the rate and monotony of magnetic flux increase, and the orientation of the axis connecting opposite polarity poles with regard to the line of sight) was not taken into account. 
a) Emerging active regions

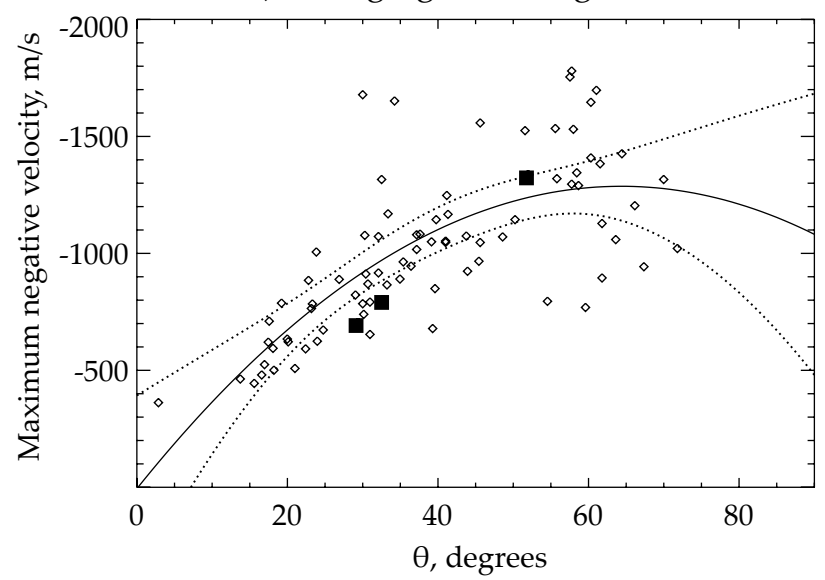

b) Quiet Sun

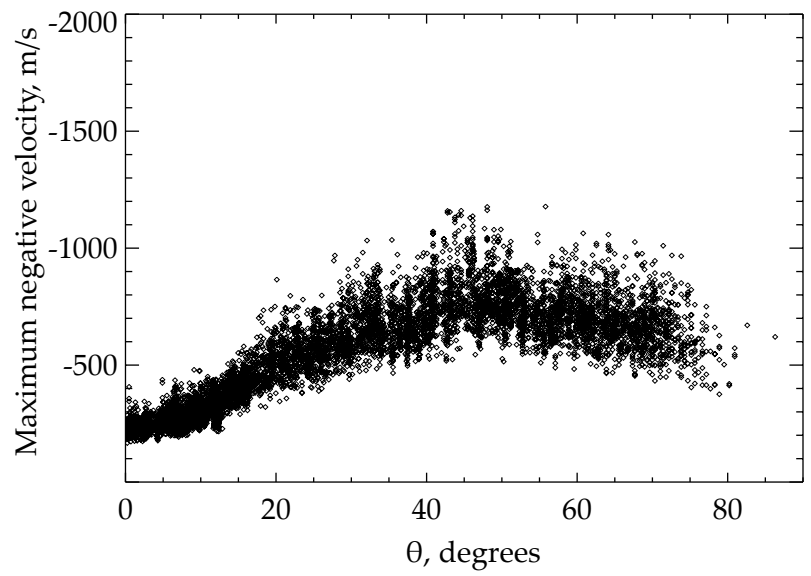

Fig. 3. a) Maximum negative velocities during the first $12 \mathrm{~h}$ of the emergence of active regions versus the heliocentric angle that corresponds to the position of the active regions at this point in time. Diamonds mark normal active regions; black squares mark reverse ones. The solid line corresponds to the nonlinear regress Eq. (2); dotted lines correspond to $99 \%$ confidence intervals for the means. b) Maximum negative velocities of convective flows in the quiet Sun versus the heliocentric angle.

\section{Results}

Figure 3a presents the maximum negative velocities observed during the first $12 \mathrm{~h}$ emergence of active regions according to their position with regard to the disk center. The distance from the disk center is expressed in the heliocentric angle $\theta-$ it is the angle between the normal to the surface and the line-ofsight to the emerging magnetic flux. The maximum negative velocities obtained are observed, as a rule, on the polarity inversion line of the emerging magnetic fields; however, they are related to the convection in adjacent regions for some events in the central part of the disk $\left(\theta<25^{\circ}\right)$. Figure 3 a shows that values of maximum negative velocities increase nonlinearly with the heliocentric angle. The mean velocity for $\theta=20^{\circ}$ is $-673 \mathrm{~m} \mathrm{~s}^{-1}$; the deviation from the mean does not exceed $200 \mathrm{~m} \mathrm{~s}^{-1}$. The mean velocity for $\theta=60^{\circ}$ is $-1277 \mathrm{~m} \mathrm{~s}^{-1}$; the deviation from the mean reaches up to $550 \mathrm{~m} \mathrm{~s}^{-1}$. One can see a growth tendency of deviation from mean with increasing $\theta$ (Fig. 3a). The high deviation for large $\theta$ indicates the existence of other relations that determe the value of the horizontal velocity of plasma flow at the site of emerging magnetic fields at the photospheric level. Our statistics include three reverse active regions whose parameters do not deviate from the dependence formed by normal active regions. Thus we see that the horizontal velocities of plasma outflows exceed the vertical ones of plasma upflows at the beginning of the emergence of active regions.

Figure $3 b$ shows the maximum negative velocities for the quiet Sun connected with convective flows. To calculate this dependence, the region on the surface was traced taking into account solar rotation. The region with a size of $40^{\prime \prime} \times 40^{\prime \prime}$ under study was going through the longitude range of W00-W67 at the latitude of S04 from 3 to 8 May 1999. The tilt of the solar North rotational axis toward the observer $B_{0}$ was $-3.5^{\circ}$. Data with a one-minute resolution were used; they were processed with the technique described in Sect. 2 of this paper. In Fig. 3b one can see that the velocities increase with the heliocentric angle; these values do not exceed $1200 \mathrm{~m} \mathrm{~s}^{-1}$. The comparison between the plots in Figs. $3 \mathrm{a}$ and $3 \mathrm{~b}$ has revealed that the horizontal velocity component at the sites of the emergence of active regions is higher than that of the convective flow in the quiet Sun.
We performed the regression analysis with the second degree polynom. As a result, we obtained the equation

$v=6.01-40.18 \theta+0.31 \theta^{2}$,

where $v$ is the maximum negative Doppler velocity during the first $12 \mathrm{~h}$ of the emergence of active regions in $\mathrm{m} \mathrm{s}^{-1}$ and $\theta$ is the heliocentric angle in degrees. According to the F-statistic this regression model is significant. The correlation ratio (estimate of the closeness of the nonlinear relation) between the value of the maximum negative velocity and the heliocentric angle $\theta$ (or the angle of view to the emerging magnetic flux) is equal to -0.74 ; this implies a high relation between the parameters under consideration. The confidence intervals for the means were calculated with a confidence probability of $99 \%$. They are marked by dotted lines in Fig. 3a. The confidence intervals have close limits for $10^{\circ}<\theta<70^{\circ}$ with high statistics and show that Eq. (2) approximates the data well. The limits of the confidence intervals essentially increase for $\theta<10^{\circ}$ and $70^{\circ}<\theta$ with low statistics. It follows from Eq. (2) that the mean vertical velocity for $\theta=0^{\circ}$ is $6 \mathrm{~m} \mathrm{~s}^{-1}$ with a confidence interval of $\pm 399 \mathrm{~m} \mathrm{~s}^{-1}$. Positive velocity values are false in this confidence range. Obviously, velocities corresponding to $\theta=0^{\circ}$ at the sites of emerging active regions will be not lower than the velocities of convective flows of the quiet Sun $\left(\sim-150 \mathrm{~m} \mathrm{~s}^{-1}\right)$. The absence of statistics for $\theta<10^{\circ}$ at the emerging active regions does not allow one to take it into account in regression Eq. (2). The mean horizontal velocity for $\theta=90^{\circ}$ from Eq. (2) is $-1081.44 \mathrm{~m} \mathrm{~s}^{-1}$ with a confidence interval $\pm 601 \mathrm{~m} \mathrm{~s}^{-1}$. The wide confidential interval shows that this value of the mean horizontal velocity is ambiguous.

A comparison of the maximum negative velocities with the total magnetic flux did not reveal any relation (Fig. 4). The comparison between the plots in Figs. 2 and 3 a also showed no connection, because high velocities were not observed in emerging active regions with high magnetic flux in the central part of the solar disk, whereas near the limb there may be high velocity values even in active regions with low magnetic flux. From the author's point of view the absence of a dependence between velocity and total magnetic flux can be explained by the fact that active region magnetic fields emerge by separate fragments on time scales from several hours to 5-7 days and therefore the maximum negative velocity during the first $12 \mathrm{~h}$ does not characterize an active region emergence as a whole.

\section{A7, page 4 of 5}


A. Khlystova: Center-limb dependence of photospheric velocities in regions of emerging magnetic fields on the Sun $(R N)$

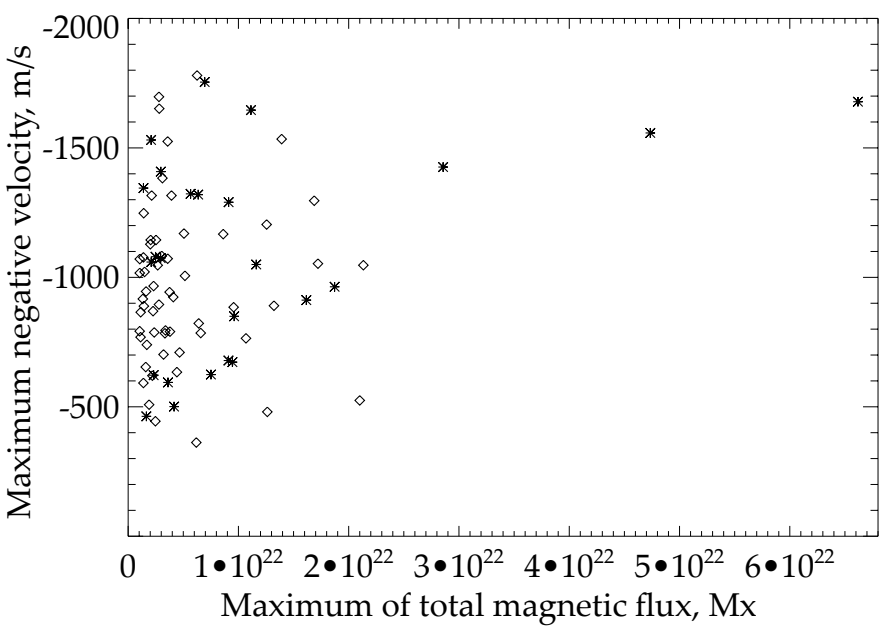

Fig. 4. Maximum negative velocities during the first $12 \mathrm{~h}$ of the magnetic field emergence versus the maximum value of the total magnetic flux in the active regions. The designations are the same as for the Fig. 2.

\section{Conclusions}

We presented a statistical study of the velocities of plasma flows that accompany the emergence of active regions during the first $12 \mathrm{~h}$ at the photospheric level with data with high temporal resolution. We found that the values of maximum negative Doppler velocities increase nonlinearly with the heliocentric angle. This shows that the horizontal velocities of plasma outflows exceed the vertical ones of plasma upflows during the first hours of active region emergence. Horizontal velocities at the sites of emerging active regions are higher than those of convective flows in the quiet Sun. This result is a direct confirmation of theoretical models that showed that magnetic fields emerging in the solar atmosphere expand faster in the horizontal direction than in the vertical direction (e.g. Shibata et al. 1989; Fan 2001; Magara \& Longcope 2003; Archontis et al. 2004).

Acknowledgements. The author is grateful to the referee for helpful comments that improved the manuscript. This work used data obtained by the SOHO/MDI instrument. SOHO is a mission of international cooperation between ESA and NASA. The Michelson Doppler Imager is a project of the Stanford-Lockheed Institute for Space Research. I am grateful to my supervisors V. M. Grigor'ev and L. V. Ermakova for their helpful suggestions on this scientific research, and to V. G. Fainshtein for very useful discussions. I would like to thank Tom Duvall and Alexander Kosovichev for discussions that helped me to understand peculiarities of SOHO/MDI Dopplergrams better. This study was supported by RFBR grants 08-02-00027-a, 09-02-00165-a, 10-02-00607-a, 10-02-00960-a, the NNSFC-RFBR grant 08-02-92211, state contract 02.740.11.0576 and by the Russian Academy of Sciences (the program 4 of the RAS Presidium and the joint project 4 of its Siberian Branch).

\section{References}

Albregtsen, F., \& Andersen, B. N. 1985, Sol. Phys., 95, 239

Archontis, V., Moreno-Insertis, F., Galsgaard, K., Hood, A., \& O’Shea, E. 2004, A\&A, 426, 1047

Bai, T., Scherrer, P. H., \& Bogart, R. S. 1998, in Structure and Dynamics of the Interior of the Sun and Sun-like Stars, ed. S. Korzennik, ESA SP-418, 607

Barth, C. S., \& Livi, S. H. B. 1990, Rev. Mex. Astron. Astrofis., 21, 549

Bruzek, A. 1967, Sol. Phys., 2, 451

Bruzek, A. 1969, Sol. Phys., 8, 29

Chang, H., Chou, D., \& Sun, M. 1999, ApJ, 526, L53

Chou, D. 1993, in The Magnetic and Velocity Fields of Solar Active Regions, ed. H. Zirin, G. Ai, \& H. Wang, ASP Conf. Ser. 46, IAU Colloq., 141, 471

Chou, D., \& Wang, H. 1987, Sol. Phys., 110, 81

Fan, Y. 2001, ApJ, 554, L111

Frazier, E. N. 1972, Sol. Phys., 26, 130

Grigor'ev, V. M., Ermakova, L. V., \& Khlystova, A. I. 2007, Astron. Lett., 33, 766

Grigor'ev, V. M., Ermakova, L. V., \& Khlystova, A. I. 2009, Astron. Rep., 53, 869

Guglielmino, S. L., Martínez Pillet, V., Ruiz Cobo, B., Zuccarello, F., \& Lites, B. W. 2006, Mem. Soc. Astron. Ital. Suppl., 9, 103

Hagenaar, H. J. 2001, ApJ, 555, 448

Harvey, K. L., \& Martin, S. F. 1973, Sol. Phys., 32, 389

Komm, R., Morita, S., Howe, R., \& Hill, F. 2008, ApJ, 672, 1254

Komm, R., Howe, R., \& Hill, F. 2009, Sol. Phys., 258, 13

Kosovichev, A. G. 2009, Space Sci. Rev., 144, 175

Kosovichev, A. G., Duvall, Jr., T. L., \& Scherrer, P. H. 2000, Sol. Phys., 192, 159

Kubo, M., Shimizu, T., \& Lites, B. W. 2003, ApJ, 595, 465

Lites, B. W., Skumanich, A., \& Martinez Pillet, V. 1998, A\&A, 333, 1053

Liu, Y., Norton, A. A., \& Scherrer, P. H. 2007, Sol. Phys., 241, 185

Magara, T., \& Longcope, D. W. 2003, ApJ, 586, 630

Scherrer, P. H., Bogart, R. S., Bush, R. I., et al. 1995, Sol. Phys., 162, 129

Schoolman, S. A. 1973, Sol. Phys., 32, 379

Shibata, K., Tajima, T., Steinolfson, R. S., \& Matsumoto, R. 1989, ApJ, 345, 584 Snodgrass, H. B. 1983, ApJ, 270, 288

Strous, L. H., \& Zwaan, C. 1999, ApJ, 527, 435

Tarbell, T., Ferguson, S., Frank, Z., et al. 1990, in Solar Photosphere: Structure, Convection, and Magnetic Fields, ed. J. O. Stenflo, IAU Symp., 138, 147

Ulrich, R. K., Bertello, L., Boyden, J. E., \& Webster, L. 2009, Sol. Phys., 255, 53

Zharkov, S., \& Thompson, M. J. 2008, Sol. Phys., 251, 369 